\title{
Erratum to: The Rho kinase inhibitor, fasudil, ameliorates diabetes-induced cardiac dysfunction by improving calcium clearance and actin remodeling
}

\author{
Dongwu Lai ${ }^{1}$. Jing $\mathrm{Gao}^{1} \cdot \mathrm{Xukun} \mathrm{Bi}^{1} \cdot \mathrm{Hong} \mathrm{He}^{1} \cdot \mathrm{Xiaolu} \mathrm{Shi}^{2} \cdot$ Shaoxiang Weng ${ }^{1}$.

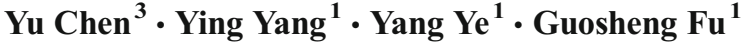

Published online: 22 September 2016

(C) Springer-Verlag Berlin Heidelberg 2016

\section{Erratum to: J Mol Med}

DOI 10.1007/s00109-016-1469-1

The original version of this article contains two mistakes on the title page; the key messages were erroneously duplicated and appended to the abstract. One keyword was misspelled. The corrected abstract and keywords are given below.

Abstract: Previous study showed inhibition of RhoA and Rho kinase (ROCK) activity with fasudil could alleviate diabetesinduced cardiac dysfunction partially due to improvement of myocardial fibrosis. However, the effect of fasudil on intracellular calcium cycling and actin remodeling, both of which are important to regulate excitation-contract coupling, is still not fully elucidated. In this study, a diabetic cardiomyopathy model was induced by a single intraperitoneal injection of streptozotocin (STZ) in male Sprague Dawley rats. Diabetic rats were treated with fasudil or placebo for 8 weeks. We

The online version of the original article can be found at http://dx.doi. org/10.1007/s00109-016-1469-1.

Guosheng Fu

fugs163@163.com

Dongwu Lai

ldw.zju@gmail.com

1 Department of Cardiology, Sir Run Run Shaw Hospital, Zhejiang University School of Medicine, 3 East Qingchun Road, Hangzhou, Zhejiang Province 310016, China

2 Experimental Research Center, China Academy of Chinese Medical Sciences, Beijing, China

3 Institute of Translational Medicine, Zhejiang University School of Medicine, Hangzhou, China found that long-term administration of fasudil, a specific Rho kinase inhibitor, significantly ameliorated diabetesinduced contractile dysfunction both at cellular and whole organ levels. Fasudil-treated rats displayed improved diastolic intracellular calcium $\left(\left[\mathrm{Ca}^{2+}\right]_{\mathrm{i}}\right)$ removal and rescued expression of protein responsible for $\left[\mathrm{Ca}^{2+}\right]_{i}$ clearance. Furthermore, our study indicated that fasudil treatment normalized the phosphorylation of the $\mathrm{PKC} \beta_{2} /$ Akt pathway in the diabetic heart, which might be the underlying mechanism accounting for the protective effect of fasudil on $\left[\mathrm{Ca}^{2+}\right]_{\mathrm{i}}$ clearance. In addition, compared to the diabetes group, fasudil also normalized the $\mathrm{G} /$ Factin ratio by preventing cofilin phosphorylation and promoted F-actin organization, suggesting a beneficial effect on actin remodeling. These findings indicate the protective effect of fasudil against diabetes-induced cardiac dysfunction via modulation of $\mathrm{Ca}^{2+}$ handling and actin remodeling.

Key words:

Rho kinase

Diabetic cardiomyopathy

Fasudil

Calcium transient

Actin remodeling 